\title{
Foliar Resistance to Phytophthora palmivora as an Indicator of Pod Resistance in Theobroma cacao
}

\author{
A. D. Iwaro, T. N. Sreenivasan, Cocoa Research Unit, and P. Umaharan, Department of Plant Science, The Uni- \\ versity of the West Indies, St. Augustine, Trinidad and Tobago, West Indies
}

\begin{abstract}
Iwaro, A. D., Sreenivasan, T. N., and Umaharan, P. 1997. Foliar resistance to Phytophthora palmivora as an indicator of pod resistance in Theobroma cacao. Plant Dis. 81:619-624.

Resistance to Phytophthora palmivora was assessed in leaves and pods of 12 diverse cacao genotypes at the penetration and post-penetration stages of infection to determine the relationship of resistance between stages and sites of infection. Penetration resistance was based on the frequency of lesions, whereas lesion size was used as a measure of post-penetration resistance. We observed significant clonal differences for leaf and pod resistance at the two stages of infection. A poor correlation between resistance at the penetration and post-penetration stages in both leaves and pods indicated that these two forms of resistance are independent and governed by different mechanisms. Correlation between resistance of leaves and pods was not significant at the penetration stage, whereas that of post-penetration resistance was positive and significant. This shows that internal or tissue resistance is common between leaf and pod and indicates that leaf resistance at the post-penetration stage of infection could be used to predict pod resistance; however, resistance of leaves at the penetration stage cannot be used to predict penetration resistance in pods. A high positive correlation obtained between attached leaves and pods with their detached counterparts showed that detached organs can be used for the prediction of resistance in attached leaves and pods.
\end{abstract}

Additional keywords: black pod, screening methods

Phytophthora palmivora occurs in all cacao production areas (10). It attacks all of the major organs of cacao, including pods, stems, leaves, and roots (7). Loss due to black pod disease alone is estimated at about $20-30 \%$ of annual cocoa production (17). Damage due to leaf, stem, and root infections by $P$. palmivora are difficult to estimate, but their effects on the health and productivity of cacao trees are known to be significant $(14,17,18)$.

Several methods are adopted by farmers to control diseases caused by Phytophthora species in cacao. The most common is the use of copper-based fungicides. Although this is reasonably effective, the high cost of chemical control poses a serious challenge to peasant farmers who produce over $50 \%$ of the worldwide production $(2,25)$. Genetic resistance has been advocated as the most effective alternative; however, progress in breeding has been hampered by a lack of effective screening methods, the diversity of Phytophthora species causing disease, and inadequate information on the types and components of resistance $(6,12)$. Cacao is a perennial plant that takes a long

Corresponding author: A. D. Iwaro

E-mail: umac0011@centre.uwi.tt

Accepted for publication 21 February 1997.

Publication no. D-1997-0326-06R

(C) 1997 The American Phytopathological Society time to breed. Consequently, tests at the seedling stage that provide information about pod resistance are earnestly sought to save time and cost. Wheeler (26) emphasized the need to understand the relationship between pod and leaf resistance to $P$. palmivora to expedite the screening of germ plasm.

In this study, experiments were conducted to assess resistance of cacao at the penetration and post-penetration stages of infection, elucidate the association between these two forms of resistance, and to determine the relationship between pod and leaf reaction to $P$. palmivora.

\section{MATERIALS AND METHODS}

The 12 cacao clones selected for this study (Table 1) represent two of the major types of cacao (Forastero and Trinitario) planted in most cacao production regions. Each of the clones were represented by 15 trees at the International Cocoa Genebank in Trinidad (ICGT). Fully-expanded green leaves of new flush (interflush 2) as described by Greathouse et al. (9) and mature unripe pods of sizes similar to those of ripe ones were used as test samples.

An isolate of Phytophthora sp. was obtained from a naturally infected cacao pod from a field at the University of the West Indies, Trinidad. Based on the characteristic "seaweed" odor of the infected pod, growth of the isolate on a $20 \%$ V8 juicecalcium carbonate agar medium and sporangial shape, size, and pedicel length, the organism was identified as $P$. palmivora. A zoospore suspension was prepared from a 10-day-old culture of the isolate (13). The concentration of zoospores was determined with a hemacytometer and adjusted to the required inoculum strength for each experiment. Leaf and pod resistance at the penetration stage of infection were assessed with the tissue-paper mount and the multiple-point methods of inoculation on leaf and pod, respectively. The number of established lesions with minimum size of $0.4 \mathrm{~mm}$ diameter on leaf and $2 \mathrm{~mm}$ diameter on pod was used as an indication of penetration resistance.

For the tissue-paper-mount inoculation, a 30- $\mu$ l drop of zoospore suspension was placed on the leaf surface with a micropipette. A $1-\mathrm{cm}^{2}$ piece of tissue paper 0.23 $\mathrm{mm}$ thick was placed on the drop of inoculum to allow a uniform spread of the zoospore suspension within the area covered by the tissue paper. Both the adaxial and abaxial leaf surfaces were inoculated on separate leaves with suspensions of 400,000 and 150,000 zoospores per $\mathrm{ml}$, respectively. These two concentrations were based on a previous serial dilution test (11). The 24 treatment combinations (12 clones, two leaf surfaces) were arranged in a completely randomized design with seven replications and incubated at $25^{\circ} \mathrm{C}$ in plastic trays lined with moist paper towel and covered with a polyethylene film. Two leaves were treated as the control for each clone with sterile distilled water used in place of a zoospore suspension. Records were kept on the number of lesions formed after a 72-h incubation period. The experiment was repeated twice.

The multiple-point inoculation was performed on the pod surface, in which 4- $\mu$ l drops of inoculum were placed at 10 points along the ridges with a micropipette. A distance of about $3 \mathrm{~cm}$ was maintained between inoculated points to avoid merging of adjacent lesions. A zoospore concentration of 30,000 per $\mathrm{ml}$ was used based on a previous serial dilution test (11). The 12 clones were replicated five times with appropriate controls and arranged in a completely randomized design. The control treatments received sterile distilled water in place of the zoospore suspension. The pods were incubated at $25^{\circ} \mathrm{C}$ in plastic trays lined with moist paper towels, covered with another plastic tray and enclosed in a polyethylene bag. After $72 \mathrm{~h}$, pods 
were assessed for the number of established lesions. Four trials were conducted.

Assessment of leaf and pod resistance at the post-penetration stage of infection was based on punch and stab inoculation methods, respectively. The spread of lesion or area of lesion formed was used as indication of post-penetration resistance. For the punch inoculation, a 4-mm-diameter hole was punched in the leaf lamina and the hole was covered at the adaxial surface with a spot plaster. The hole in the abaxial surface was filled with a 4-mm-diameter filter paper disk previously immersed in a 200,000 zoospores per $\mathrm{ml}$ suspension. A control leaf from each clone was treated with sterile distilled water in place of the zoospore suspension. Inoculated leaves were arranged in a completely randomized design with five replicates per clone and incubated at $25^{\circ} \mathrm{C}$ in trays lined with moist tissue paper and covered with a polyethylene film. After incubation for $144 \mathrm{~h}$, leaves were assessed for lesion size with a leaf area meter (MK2, Delta $\mathrm{T}$ Services by Burnwell, Cambridge, England). The experiment was repeated twice.

For the stab inoculation, a standard injury $0.3 \mathrm{~cm}^{2}$ was created on the pod surface with an apparatus that consisted of 20 3 -mm pins. The wounded spot was inoculated with a 4-mm-diameter filter paper disk previously immersed in a 200,000 zoospores per $\mathrm{ml}$ suspension and covered with a spot plaster. This concentration of inoculum was reported as optimum in similar inoculations conducted by Sreenivasan (23), Sitapai (19), and Okey (16). A control pod from each clone was treated with sterile distilled water in the place of the zoospore suspension. Inoculated pods were arranged in a completely randomized design with five replicates per clone and incubated at $25^{\circ} \mathrm{C}$ in trays lined with moist tissue paper and covered with a polyethyl- ene film. After incubation for $120 \mathrm{~h}$, the size of established lesion was determined from brown paper cutouts trimmed to the size of each lesion and measured with a
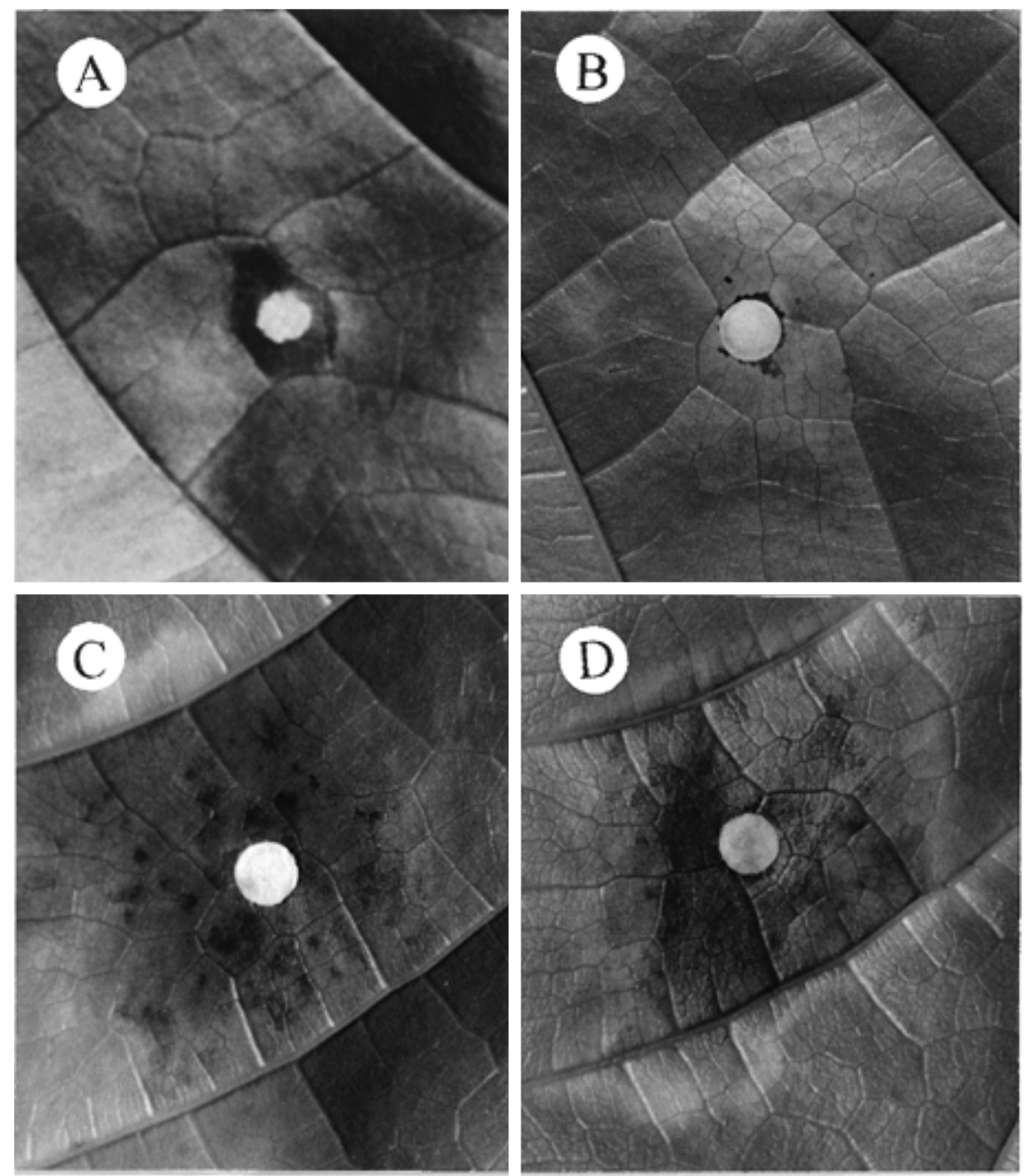

Fig. 1. Pattern of spread of lesions on mature leaves of cacao clones (A) SCA 6, (B) Catongo, (C) ICS 8, and (D) ICS 1 inoculated with Phytophthora palmivora. leaf area meter. The experiment was repeated twice.

Comparisons of reactions of attached leaves and pods with their detached coun-

Table 1. Lesion number and size on leaves and pods $^{\mathrm{x}}$ of 12 cacao clones after inoculation with Phytophthora palmivora

\begin{tabular}{|c|c|c|c|c|c|c|c|c|c|c|}
\hline \multirow[b]{3}{*}{ Clones } & \multicolumn{6}{|c|}{ Number of lesions (mean) } & \multicolumn{4}{|c|}{ Lesion size, cm $^{2}$ (mean) } \\
\hline & \multicolumn{2}{|c|}{ Leaf (adaxial) } & \multicolumn{2}{|c|}{ Leaf (abaxial) } & \multicolumn{2}{|c|}{ Pod } & \multicolumn{2}{|c|}{ Leaf } & \multicolumn{2}{|c|}{ Pod } \\
\hline & $\mathbf{a}^{\mathbf{y}}$ & $\mathbf{b}^{\mathbf{z}}$ & $\mathbf{a}^{\mathbf{y}}$ & $\mathbf{b}^{\mathbf{z}}$ & $\mathbf{a}^{\mathbf{y}}$ & $\mathbf{b}^{\mathbf{z}}$ & $\mathbf{a}^{\mathbf{y}}$ & $\mathbf{b}^{\mathbf{z}}$ & $\mathbf{a}^{\mathrm{y}}$ & $\mathbf{b}^{\mathbf{z}}$ \\
\hline ICS 1 & 2.65 & 6.0 & 5.35 & 27.6 & 2.35 & 5.5 & 2.45 & 5.0 & 8.27 & 68.4 \\
\hline ICS 6 & 2.37 & 4.6 & 5.14 & 25.4 & 2.85 & 8.1 & 2.17 & 3.7 & 7.41 & 54.9 \\
\hline ICS 8 & 2.45 & 5.0 & 4.09 & 24.9 & 2.47 & 6.1 & 2.51 & 5.3 & 8.54 & 73.0 \\
\hline ICS 40 & 1.70 & 1.9 & 4.37 & 18.1 & 2.47 & 6.1 & 1.73 & 2.0 & 7.11 & 50.6 \\
\hline ICS 84 & 3.24 & 9.5 & 5.07 & 24.7 & 2.39 & 5.7 & 1.76 & 2.1 & 6.97 & 48.6 \\
\hline ICS 95 & 1.84 & 2.4 & 4.99 & 23.9 & 2.83 & 8.0 & 1.82 & 2.3 & 5.91 & 34.9 \\
\hline PA 30 & 1.64 & 1.7 & 3.86 & 13.9 & 2.51 & 6.3 & 1.84 & 2.4 & 8.40 & 70.5 \\
\hline PA 46 & 2.61 & 5.8 & 3.38 & 10.4 & 2.51 & 6.3 & 1.87 & 2.5 & 7.46 & 55.6 \\
\hline SCA 6 & 2.26 & 4.1 & 3.79 & 13.4 & 2.24 & 5.0 & 1.45 & 1.1 & 5.77 & 33.3 \\
\hline SCA 12 & 2.02 & 3.1 & 3.48 & 11.1 & 2.85 & 8.1 & 1.70 & 1.9 & 7.08 & 50.1 \\
\hline IMC 67 & 1.97 & 2.9 & 3.94 & 14.5 & 2.86 & 8.2 & 2.28 & 4.2 & 8.88 & 78.8 \\
\hline CATONGO & 2.05 & 3.2 & 2.98 & 7.9 & 2.83 & 8.0 & 2.26 & 4.1 & 8.82 & 77.8 \\
\hline $\mathrm{LSD}_{0.05}$ & 0.41 & & 0.53 & & 0.14 & & 0.14 & & 0.40 & \\
\hline
\end{tabular}

${ }^{\mathrm{x}}$ Means were based on 14 and 20 replicates for lesion number on leaves and pods respectively. Lesion sizes were assessed on 10 replicates in both organs.

Tissue-paper-mount and multiple point methods were used to assess lesion number, while lesion sizes were determined following punch and stab inoculation methods in leaves and pods, respectively.

y Mean of transformed data $\sqrt{ }(x+1)$.

${ }^{\mathrm{z}}$ Mean of transformed data $\sqrt{ } x$. 
terparts to inoculation were based on postpenetration tests for resistance only. Tests of penetration resistance based on the tissue-paper-mount and multiple-point inoculations were difficult to perform on attached leaves and pods and were not pursued.

Twenty-five cacao seedlings from a segregating population in the greenhouse were used for a comparison of attached and detached leaves. Two leaves detached from each seedling were inoculated in the laboratory with the punch method described earlier, while two attached leaves were inoculated on each seedling in the greenhouse. The attached leaves on each plant were enclosed in a polyethylene bag containing a moist paper towel to maintain high humidity within the bag. The size of lesion in both detached and attached leaves was assessed after 6 days.

Ten clones located in the St. Augustine cocoa field were used in the comparison of attached and detached pods. Five mature pods were harvested from each clone and inoculated in the laboratory with the stab method of inoculation, while another five undetached pods were inoculated per clone by the same method and the same zoospore suspension in the field. The attached pods on each plant were enclosed in polyethylene bags containing a moist paper towel to maintain high humidity within the bag. After 5 days, all of the inoculated pods, both in the laboratory and field, were assessed for lesion size.

Data collected on the frequency and spread of lesions in leaves and pods were subjected to square root transformation. An analysis of variance was performed on the transformed data using the MINITAB statistical software to determine the significance of genotypic differences in penetration and post-penetration reactions to $P$. palmivora. In addition, the relationship between penetration and post-penetration reaction was tested by regression analysis. The correlation between leaf and pod resistance at penetration and post-penetration stages of infection was also assessed to determine whether resistance is systemic or localized with respect to these two organs. Further, data collected on detached and attached leaves and pods were subjected to correlation analysis to determine the utility of detached-organ assays in screening programs.

\section{RESULTS}

Clonal differences in leaf and pod resistance at the penetration stage of infection. There were significant differences $(P$ $<0.05)$ among some clones in their reactions to $P$. palmivora at the penetration stage, based on the number of lesions observed per square centimeter (Table 1). The number of lesions observed among the clones varied between 27.6 and 7.9 on the abaxial surface, and between 9.5 and 1.7 on the adaxial surface. The adaxial surface
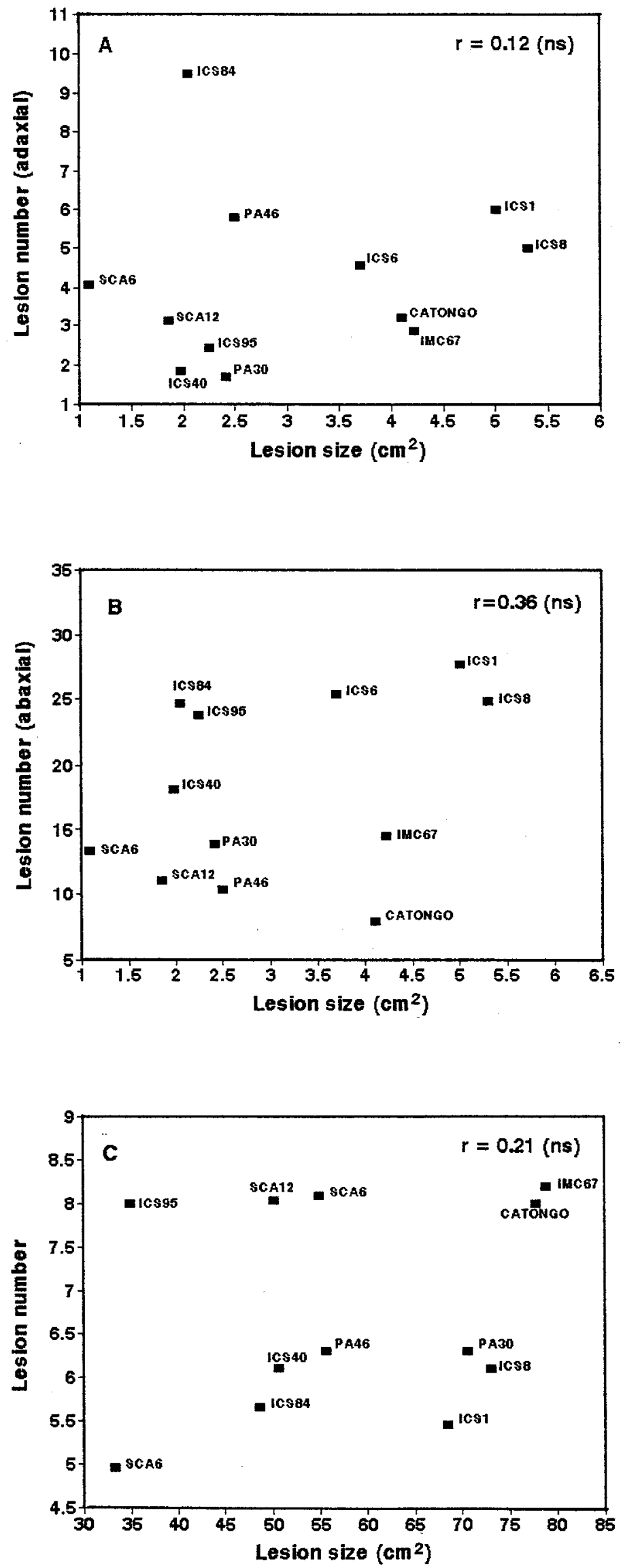

Fig. 2. Relationship between lesion number and size in (A) adaxial and (B) abaxial sides of leaves and (C) in pods of 12 cacao clones inoculated with Phytophthora palmivora (ns = not significant). 

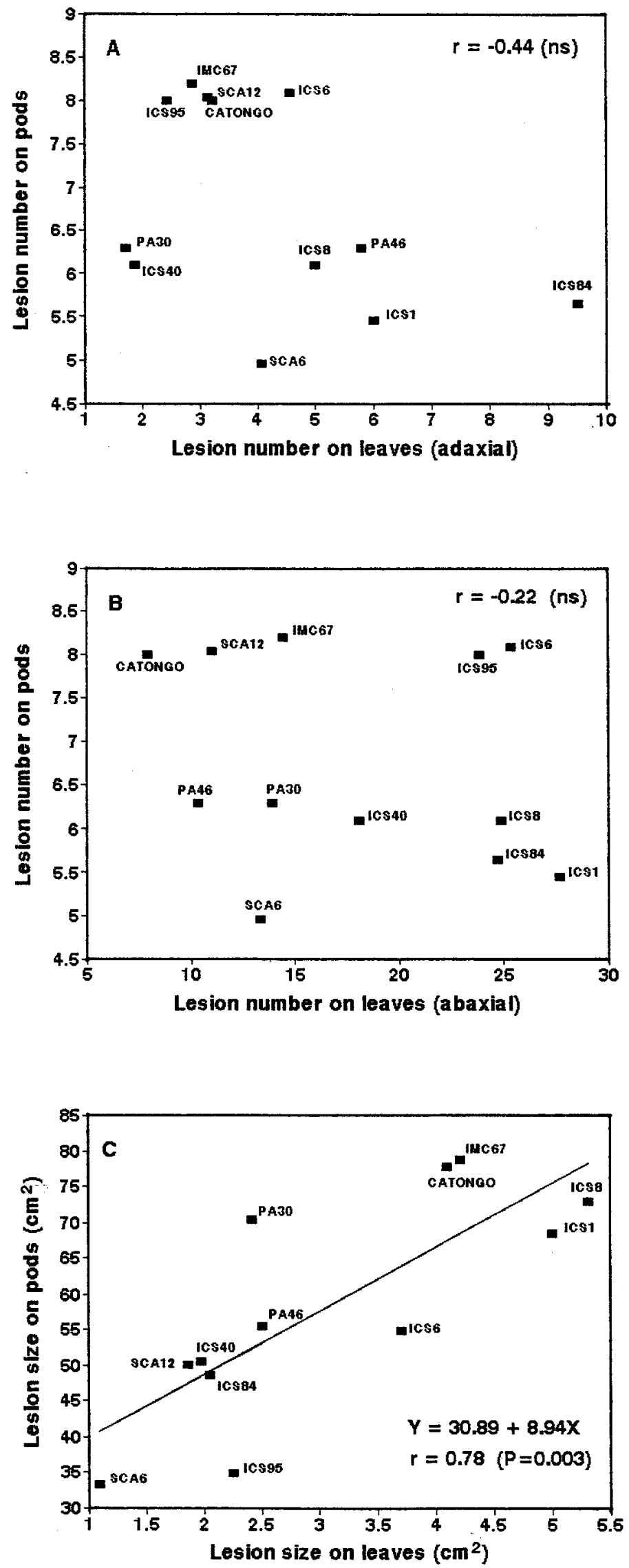

Fig. 3. Relationship between lesion number on (A) adaxial and (B) abaxial sides of leaves and on pods and between (C) lesion size on leaves and pods in 12 cacao clones inoculated with Phytophthora palmivora $(\mathrm{ns}=$ not significant). was less susceptible to infection than was the abaxial surface, although the former was inoculated with a lower zoospore concentration. Lesion numbers were relatively high on both leaf surfaces in ICS 1, ICS 6, ICS 8, and ICS 84. In ICS 95, ICS 40, and PA 46, however, lesion numbers were relatively high on one leaf surface but not on the other. Fewer lesions were recorded on both leaf surfaces for Catongo, IMC 67, SCA 12, PA 30, and SCA 6. The correlation value between resistance of the two leaf surfaces $(r=0.42)$ was moderately low, suggesting that the reaction of one surface may be difficult to predict from the other. However, because the inherent level of resistance at the adaxial surface is high, resistance at the abaxial surface is seemingly more important to protect the leaves from infection for practical purposes.

There were also significant differences $(P<0.05)$ among some clones for the number of lesions produced on inoculated pods (Table 1). The mean number of lesions produced on IMC 67, ICS 95, ICS 6 , SCA 12, and Catongo were large (8.0-8.2), but not significantly different from each other. Relatively fewer lesions (5.0-6.3) were produced on the other tested clones. Clones SCA 6, ICS 1, and ICS 84 showed the lowest lesion frequency (5.0-5.7) and appeared most promising among the clones tested for resistance at the penetration stage of infection in pods.

Clonal differences in leaf and pod resistance at the post-penetration stage of infection. Lesion sizes on leaves varied significantly $(P<0.05)$ among the clones (Table 1). SCA 6 produced the smallest lesion size. Lesion sizes were comparatively large in ICS 8, ICS 1, IMC 67, Catongo, and ICS 6, and intermediate in SCA 12, ICS 40, ICS 95, and PA 30. Four distinct patterns of spread were observed among the clones (Fig. 1A to D). In clones with relatively small lesions, such as SCA 6 , necrosis was restricted predominantly within the mesophyll (Fig. 1A). In contrary, the spread of lesions through the veins was in advance of the mesophyll necrosis in Catongo producing large lesions (Fig. 1B). Distal lesions, which appeared as discrete spots away from the primary lesion, were observed in ICS 8 (Fig. 1C). Rapid spread of lesions through both mesophyll and veins was observed in ICS 1, resulting in very large lesions (Fig. 1D).

A significant difference $(P<0.05)$ was also observed among clones for pod resistance to pathogen invasion based on lesion size (Table 1). Relatively smaller lesions were recorded for SCA 6 and ICS 95. Lesion sizes for ICS 6, ICS 40, ICS 84, SCA 12 , and PA 46 were moderate, whereas relatively large lesions were produced on other clones.

Relationship between penetration and post-penetration resistance in leaf and pod. A poor correlation was obtained be- 
tween the reaction of leaves at the penetration and post-penetration stages of infection (Fig. 2A and B). Similarly, the correlation was poor between pod reaction to penetration and post-penetration (Fig. 2C). These two forms of resistance are apparently independent and are likely governed by different mechanisms. The correlation analysis performed between leaf and pod reactions at the penetration stage did not show any significant relationship between the two organs (Fig. 3A and B). In the contrary, a high positive correlation was obtained between leaf and pod for postpenetration resistance (Fig. 3C). This relationship shows that internal or tissue resistance is likely common to leaf and pod.

Comparison between the reaction of detached and attached leaves and pods to $P$. palmivora infection. A high positive correlation was obtained between attached and detached leaves (Fig. 4A), suggesting that detached leaves can be used for the prediction of resistance in attached leaves. A similar relationship was observed between detached and attached pods (Fig. 4B). However, susceptibility was higher in detached leaves and pods than in the attached organs.

\section{DISCUSSION}

Agrios (1) pointed out that resistance could be effective from the point of entry of the pathogen (penetration) or at a later stage during its development within the host tissue (post-penetration). In this study, different cacao clones were found to show varying degrees of susceptibility at the penetration and post-penetration stages of the infection process in both leaves and pods. Such differential responses of clones to method of inoculation suggest that the resistance mechanisms operative at the penetration and post-penetration stages of infection may be distinct. Poor correlations obtained between resistance at the penetration and post-penetration stages of infection, in both leaves and pods, also clearly show that they are independent. Previous investigations had shown that stomata on cacao leaves could serve as infection courts to Crinipellis perniciosa, the pathogen of witches'-broom disease $(8,24)$ and more recently to $P$. palmivora (11). The influence of cuticle thickness, stomatal frequency, and size on resistance to penetration by some pathogens also has been reported in sweet potato, poplar, and water yam $(5,22,15)$. It is possible that some of these factors could be responsible for differences in clonal reaction of leaf and pod to infection at the penetration stage. The differences among clones at the postpenetration level as shown in sizes and patterns of spread of lesions could be due to some biochemical factors. Spence $(20,21)$ indicated the involvement of oxidizable phenols and polyphenol oxidase activity in determining lesion size in cacao. Although the factors responsible for leaf and pod resistance to $P$. palmivora are yet to be characterized fully, it is evident that there are two levels of resistance in the two organs investigated. The implication of this is that selection could be made independently for penetration and post-penetration resistance, so that they could be combined in breeding to improve the existing levels of cacao resistance to $P$. palmivora.

Our study also showed a poor relationship between pod and leaf reaction at the penetration stage of infection. Iwaro (11) indicated that there was a poor correlation between the stomatal frequency on pods and leaves of the 12 selected clones, which may account for the poor relationship in the reaction of the two organs to infection by $P$. palmivora at the penetration stage. In contrast, a high positive correlation was obtained between pod and leaf resistance at the post-penetration stage of infection, which suggests that the mechanism conferring post-penetration resistance is systemic within leaf and pod. The benefit of this finding is that pod resistance can be predicted based on estimates of lesion size on leaves of seedlings, thus, large populations of progenies can be screened at the seedling stage with savings in time and cost. While a similar relationship has been established between pod and root $(3,4)$ and, pod and stem $(19,27,28)$, the immense value of a leaf assay in screening seedlings of a perennial crop such as cacao cannot be overemphasized. However, the lack of relationship between leaf and pod reactions
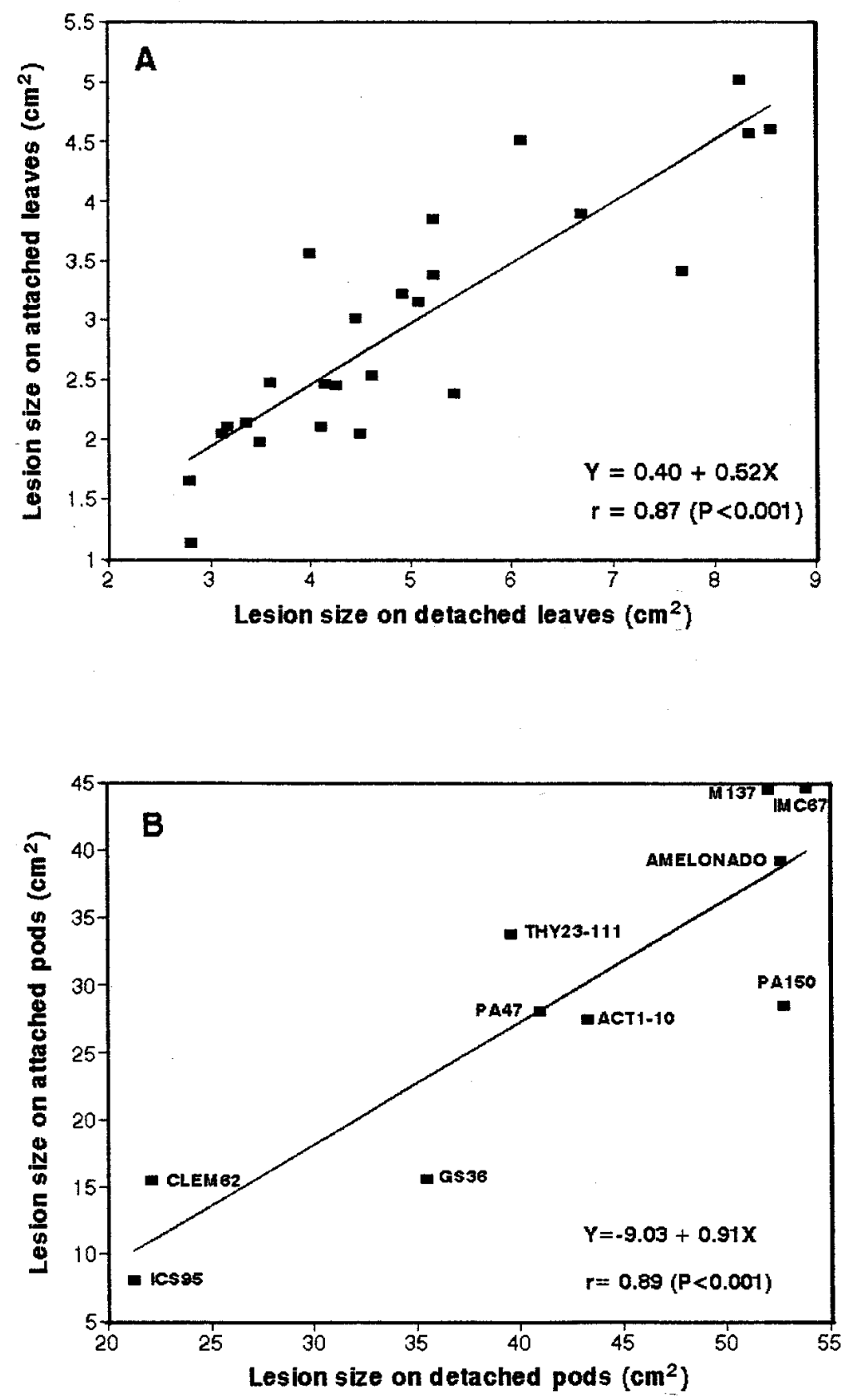

Fig. 4. Relationship between the reactions of detached and attached (A) leaves and (B) pods of Theobroma cacao inoculated with Phytophthora palmivora. 
at the penetration stage of infection suggests that selection for this component of resistance should be delayed to the podbearing stage. The benefit of such a twostage selection process is that the large segregating population could be reduced in size based on a initial leaf screen for postpenetration resistance and only resistant progenies could be retained to assess penetration resistance in pods.

The strong association between the reaction of detached and attached leaves and pods showed that a reliable prediction of leaf and pod resistance to $P$. palmivora infection can be made from laboratory inoculations of detached leaves and pods. Laboratory inoculations are more convenient and, in general, provide the opportunity to screen for resistance under standard conditions that limit the chances of escape, making them more rapid and reliable. A selected subset based on laboratory tests could be used in field tests to verify results. The fact that the reactions of detached and attached leaves and pods to infection by $P$. palmivora are closely associated also confirms the effectiveness of the inoculation methods and the possibility of using them for screening in future breeding programs aimed at improving the resistance of cacao to Phytophthora infection. Lack of Phytophthora species $\times$ genotype interactions in a preliminary test involving $P$. palmivora and $P$. capsici (not reported in this study), and the additive nature of resistance $(11,19,25)$ suggest that genetic resistance derived from breeding programs of this kind would have wider acceptance.

\section{ACKNOWLEDGMENTS}

We thank the European Economic Community (EEC) and the Biscuit, Cake, Chocolate and Confectionery Alliance (BCCCA) for financial support, and J. A. Spence and R. A. Lass for their encouragement and support.

\section{LITERATURE CITED}

1. Agrios, G. N. 1988. Plant Pathology. 3rd ed. Academic Press, San Diego, California.
2. Amponsah, J. D. 1988. Studies on field resistance of cocoa varieties to $P$. palmivora (Butl.) Butl. infection. Pages 557-567 in: Proc. Int. Cocoa Res. Conf., 10th.

3. Asomaning, E. J. A. 1964. Root infection of cocoa by $P$. palmivora. Cocoa Res. Inst. Ghana, 1962-63 Rep. 23-25.

4. Asomaning, E. J. A. 1964. Varietal resistance of young clones and seedlings of cocoa (Theobroma cacao L.) to root infection by $P$. palmivora. Trop. Agric. (Trinidad) 41:251256.

5. Bajit, G. B., and Gapasin, R. M. 1987. Relationship between morphological characteristics and varietal resistance of sweet potato to Scab infection caused by Sphaceloma batatas. Ann. Trop. Res. 9:75-83.

6. Bartley, B. G. D. 1986. Cacao (Theobroma cacao L.). Pages 25-42 in: Breeding for durable resistance in perennial crops. FAO, Rome.

7. Firman, I. D. 1974. Cocoa canker. Pages 131140 in: Phytophthora Disease of Cocoa. P. H. Gregory, ed. Longman, London.

8. Frias, G. A. 1987. An inoculation method to evaluate resistance to Witches' broom disease of cacao. Ph.D. thesis. University of Florida, Florida.

9. Greathouse, D. C., Laetsch, W. M., and Phinney, B. O. 1971. The shoot-growth rhythm of a tropical tree (Theobroma cacao L.). Am. J. Bot. 58(4):281-286.

10. Gregory, P. H. 1974. Preface. Pages v-vi in: Phytophthora Disease of Cocoa. P. H. Gregory, ed. Longman, London.

11. Iwaro, A. D. 1995. Mechanisms of resistance to Phytophthora palmivora (Butler) Butler in cacao (Theobroma cacao L.) and their genetic basis. Ph.D. thesis. The University of the West Indies, Trinidad and Tobago.

12. Kennedy, A. J., Lockwood, G., Mossu, G., Simmonds, N. W., and Tan, G. Y., 1987. Cocoa breeding: past, present and future. Cocoa Grow. Bull. 38:5-22.

13. Lawrence, J. S. 1978. Evaluation of methods for assessing resistance of cacao (Theobroma cacao L.) cultivars and hybrids to Phytophthora palmivora (Butler) Butler. Bol. Tec. 62: $46 \mathrm{pp}$.

14. Manco, G. R. 1974. Blight of shoots, chupons, cuttings, grafts, and seedlings. Pages 149-152 in: Phytophthora Disease of Cocoa. P. H. Gregory, ed. Longman, London.

15. Nwankiti, A. O., and Okpala, E. U. 1984. Source of resistance to anthracnose/blotch disease of water yam (Dioscorea alata) caused by Colletotrichum gloeosporioides Penz. Beitr. Trop. Landwirtsch. Veterinarmed. 4:401-406.
16. Okey, E. N. 1996. Histopathological and biochemical studies on cacao (Theobroma cacao L.) canker caused by Phytophthora palmivora (Butler) Butler. Ph.D. thesis. The University of the West Indies, Trinidad and Tobago.

17. Opeke, L. K., and Gorenz, A. M. 1974. Phytophthora pod rot; symptoms and economic importance. Pages 117-124 in: Phytophthora Disease of Cocoa. P. H. Gregory, ed. Longman, London.

18. Prior, C. 1981. Phytophthora bark canker of cocoa in Papua New Guinea. Pages 272-275 in: Proc. Int. Cocoa Res. Conf., 7th.

19. Sitapai, E. C. 1989. Studies on the resistance in cocoa (Theobroma cacao L.) to Phy tophthora palmivora (Butler) Butler. M. Phil. thesis. The University of the West Indies. Trinidad and Tobago.

20. Spence, J. A. 1961. Black pod disease of cocoa II: A study in host-parasite relations. Ann. Appl. Biol. 49:723-734.

21. Spence, J. A. 1961. Probable mechanism of resistance of varieties of cacao to black pod disease caused by $P$. palmivora. Nature 192:278.

22. Spiers, A. G., and Hopcroft, D. H. 1984. Influence of leaf age, leaf surfaces and frequency of stomata on the susceptibility of poplar cultivars to Marssonina brunnea. Eur. J. For. Pathol. 14:270-282.

23. Sreenivasan, T. N. 1985. A rapid method of inoculation of Phytophthora palmivora (Butler) Butler on attached pods. Pages 293-302 in: Proc. Int. Cocoa Res. Conf., 9 th.

24. Sreenivasan, T. N., and Dabydeen, S. 1989. Modes of penetration of young cocoa leaves by Crinipellis perniciosa. Plant Dis. 73:478481.

25. Tan, G. Y., and Tan, W. K. 1990. Additive inheritance of resistance to pod rot caused by $P$. palmivora in cocoa. Theor. Appl. Genet. 80:258-264.

26. Wheeler, B. E. J. 1992. Assessment of resistance to major cocoa diseases. Pages 139-145 in: Proc. Int. Workshop on Conservation, Characterization and Cocoa Genetic Resources in the 21st Century. Cocoa Res. Unit, University of the West Indies, Trinidad and Tobago.

27. Zentmyer, G. A. 1969. Resistance of cacao to $P$. palmivora. Pages 147-148 in: Proc. Int. Cocoa Res. Conf., 2nd.

28. Zentmyer, G. A., Mircetich, S. M., and Mitchell, D. M. 1968. Tests for resistance of cacao to $P$. palmivora. Plant. Dis. Rep. 52:790-791. 